\title{
El Estado y las garantías constitucionales: formación de cárcavas en las colonias de San Salvador
}

\section{The State and the constitutional guarantees: gully formation in San Salvador neighborhoods}

\author{
Carolina Lucero Morán \\ Universidad Tecnológica de El Salvador \\ Carolina.moran@utec.edu.sv \\ Recibido: 1/04/18- Aceptado: 11/08/18
}

URI: http://hdl.handle.net/11298/455

DOI: http://dx.doi.org/10.5377/entorno.v0i65.6052

\section{Resumen}

La investigación tuvo por objetivo determinar el cumplimiento de las garantías constitucionales que se presentan en el fenómeno de la formación de cárcavas. El estudio fue realizado en las colonias más vulnerables de San Salvador y Soyapango, las que incluyeron Málaga, Las Cañas, Las Victorias y Altos del Boulevard, dentro de las cuales se entrevistó a 100 participantes, hombres y mujeres, de edades de 18 a 50 años, residentes de dichas colonias, dueños de vivienda y arrendatarios. El estudio fue de tipo exploratorio descriptivo (Montero y León, 2007), con una muestra estratificada, que se eligió con la fórmula para poblaciones finitas, para un nivel de confianza del $90 \%$ y un margen de error del $6 \%$. Entre los hallazgos principales se destaca que la causa más significativa de la formación de cárcavas es el fenómeno de la erosión. A su vez, se determinaron las responsabilidades del Estado en torno a este problema, entre las que se incluye la prevención para evitar el deterioro de las vías urbanas y el territorio.

\section{Abstract}

The objective of this research was to determine the compliance of constitutional guarantees that arise in the phenomenom of gully formation. This study was conducted in the most vulnerable neighborhoods of San Salvador and Soyapango, which included Málaga, Las Cañas, Las Victorias and Altos del Boulevard; there, a total of 100 participants-men and women-ages 18 to 50, residents of said neighborhoods either as home owners or tenants, were interviewed.

The study was exploratory-descriptive (Montero y León, 2007), with a stratified sample that was chosen with the formula for finite populations with a confidence level of $90 \%$ and a margin of error of $6 \%$. Among the main findings stands out the fact that the most significant cause in gully formation is erosion. At the same time, the State responsibilities around this problem were determined; said responsibilities include prevention, which can avoid the deterioration of urban roads. 


\section{Palabras clave}

Cárcavas. Erosión de suelos. Conservación de suelos. Estructura de suelos. Composición de suelos. Garantías constitucionales.

\section{Keywords}

Gullies. Soil erosion. Soil preservation. Soil structure. Soil composition. Constitutional guarantees.
La formación de cárcavas en El Salvador, como producto de la erosión por la deforestación y el desgaste de la tierra resultantes de las malas políticas del aparato de la construcción, han puesto sobre el lienzo del espectador salvadoreño un panorama de depresiones que amenaza con engullir a las poblaciones circundantes.

Cada vez son más las personas cuya necesidad de establecer una vivienda; $y$ formar asentamientos humanos las orienta a la tala indiscriminada de árboles. También, cada vez son más las empresas constructoras que se suman a esta tarea, con el fin de proporcionar vivienda digna a todos aquellos capaces de acceder a ella. Estas viviendas, a menudo son erigidas sobre terrenos rellenados y compactados, que antes fueron viejos cauces de ríos o pozas secas proclives, sin duda, a ser futuras cárcavas si no reciben el tratamiento adecuado.

La jurisprudencia nacional e internacional considera el acceso a la vivienda como un derecho humano de primera importancia (Ramírez, 2010). El Estado de El Salvador, en su Art. $119 \mathrm{Cn}$., establece como garantía constitucional que las personas tienen derecho a una vivienda adecuada y a ser sus propietarias; derecho que también tiene asidero en el Art. 2, que dice: "Toda persona tiene derecho a la vida, a la integridad física y moral, a la libertad, a la seguridad, al trabajo, a la propiedad y posesión, y a ser protegida en la conservación y defensa de los mismos". Este derecho de propiedad de una vivienda digna también está consagrado en muchos instrumentos internacionales, entre ellos, la Declaración Universal de Derechos Humanos y el Pacto Internacional de Derechos Económicos, Sociales y Culturales, en el ámbito de la Organización de las Naciones Unidas (ONU).

Enmarcados en este derecho, el gobierno de El Salvador ha activado diversas campañas orientadas a proporcionar ese tipo de vivienda: "Me refiero al programa de mejoramiento y construcción de vivienda de interés social que hemos denominado "Casa para todos"; y que beneficiará a 27.947 familias de las zonas urbanas y rurales del país" (Funes, 2009), que si bien han paliado la necesidad de vivienda de muchos salvadoreños, también han acrecentado el problema de la deforestación en todo el territorio.

A esto se debe agregar las construcciones de aquellos que, obligados por la pobreza, la guerra, la desintegración familiar y la migración, entre otros fenómenos, se han sumado a un desorden habitacional que refleja la insuficiencia de los gobiernos para solventar ese derecho a la vivienda digna.

Al respecto, Ramírez (2010) agrega que, "cuando la vivienda deja de ser un derecho para convertirse predominantemente en un negocio, esta queda restringida y determinada por las fuerzas especulativas y excluyentes del mercado; solo tienen posibilidad de comprar una vivienda los que son sujetos de crédito. Las familias pobres deben asentarse en zonas alejadas, carentes de servicios básicos y en viviendas precarias". El problema se agrava si —como ocurre en El Salvador- no se cuenta con una política de vivienda ni con normativas jurídicas específicas que enfrenten el problema habitacional desde un enfoque de derechos humanos.

Valladares (2011) advierte que casi la mitad de los 43 millones que habitan Belice, El Salvador, Honduras, Nicaragua, Costa Rica, Panamá y Guatemala viven en la pobreza, y, por tanto, tienen serias dificultades para acceder a una vivienda digna porque este derecho ha quedado a merced del mercado, obligando a muchos a vivir en asentamientos irregulares, hacinados y en condiciones infrahumanas o en lugares de alto riesgo, como laderas de montañas o costas de ríos que se desbordan.

En El Salvador, son muchas familias, en diversas colonias, que viven pendientes de alguna cárcava que se ha formado en su área; sus casas a veces están justo a la orilla o yacen en el fondo de esta. Si bien la Constitución de la República de El Salvador garantiza el bienestar de la gente y la Ley del Medio Ambiente ofrece la oportunidad de un medio ambiente sano en el que las personas 
puedan encontrar tutela a sus derechos fundamentales, ambas garantías son constantemente irrespetadas. A esto se agrega la poca atención que se da al articulado propuesto en las leyes penales de la República atinentes a los delitos ambientales.

La población de nuestro país vive día a día las consecuencias de una planificación urbana deficiente y una política de aprovechamiento agresivo de los pocos recursos naturales que tenemos (OMS 2008). Cada invierno, la población capitalina sufre los estragos de vivir en un medio cuyo entorno ambiental es incapaz de mantener un equilibrio de subsistencia debido al abuso mediante la deforestación, entre otros factores. De tal forma, se hace indispensable determinar a priori las causas de la formación de cárcavas para tener insumos para el diseño de políticas públicas y ambientales que consideren la relación de los habitantes y su entorno a largo plazo. El Gobierno toma medidas correctivas en cada invierno, pero no está claro hasta qué punto llega su responsabilidad en su prevención, por lo tanto, se hace necesario un estudio que aborde su dimensión jurídica.

Obviamente, tanto la tala de árboles como los efectos de la infraestructura de una superpoblación, que cada día se imponen en el paisaje, han dado como resultado un fenómeno que se ha extendido más allá de toda comprensión y toda economía, desnutriendo con ello el rostro de un El Salvador en donde los hogares ya no pueden sostenerse frente al embate de los desastres naturales que periódicamente se presentan.

Esa es actualmente la realidad de las colonias Málaga, Las Cañas, Las Victorias. y Altos del Boulevard, entre otras que sufren este deterioro.

No obstante lo anterior, "el problema no es solo del Estado. Es necesaria la participación de las comunidades y de los empresarios privados en la toma de decisiones y en la inversión; esta participación ha sido de carácter secundario, siendo por consiguiente el Estado el protagonista principal en la reforestación. Estos factores indudablemente han sido fundamentales en el fracaso de gran parte de los programas de reforestación llevados a cabo" (Arguello, 1994).

\section{Condicionantes para el desarrollo del estudio}

Para abordar las variables que el estudio pretendía medir, se planteó como objetivo general determinar el cumplimiento de las garantías constitucionales que se presentan en el fenómeno de la formación de cárcavas. Para tal fin, se establecieron como objetivos específicos el determinar las causas de estas e identificar las obligaciones jurídicas de los diferentes actores al respecto en las colonias de San Salvador.

Para la concreción de estos objetivos, se utilizó un diseño de investigación de tipo empírico descriptivo y documental, haciéndose necesario el acopio de información en campo junto con una revisión bibliográfica exhaustiva del fenómeno, utilizando la lista de cotejo y el cuestionario como instrumentos principales para la recolección de datos.

Las implicaciones teóricas del estudio están divididas en una vertiente jurídica y otra ecológica, confluyendo ambas en la rama del bienestar social. La realización de este estudio implicó la comparación de protocolos y leyes establecidos a escala internacional en el país, encontrando que no se cumplen en la medida en que deberían y que existe un divorcio entre las garantías expresadas en la Constitución de la República y la práctica social de los entes gubernamentales relacionados con el tema. Una de las garantías que más nos ocupa en esta investigación es el derecho a la vivienda digna señalado en el Art. 119 de la Constitución (Constitución de la República de El Salvador, 2011).

Las limitantes principales del estudio fueron la poca capacidad de desplazamiento hacia los sitios del estudio, la imposibilidad de acceder a algunas colonias debido a los obstáculos establecidos por la criminalidad y la consecuente peligrosidad de las cárcavas tomadas en cuenta para la realización del proyecto. La participación de los sujetos afectados fue vital, pues hizo posible conocer las experiencias de los entrevistados con la ayuda de los respectivos instrumentos.

\section{Las cárcavas: resultado de la interferencia del hombre}

La incidencia en la formación de cárcavas es un problema que día tras día crece en El Salvador. Frente a la debilidad que muestra la tierra, que se desmorona, a menudo es necesario preguntarse si las cárcavas son únicamente un problema de los que viven muy cerca de ellas, o si son un problema social y económico que debe atenderse urgentemente, por cuanto las consecuencias de su envergadura pueden 
rozar fácilmente la fatalidad, frente a la fragilidad del ser humano. Las especulaciones rápidamente toman su lugar al considerar la falta de adecuadas políticas para manejar los recursos naturales y la ausencia total de respeto - del ser humano- frente a un medio ambiente que ha sufrido de forma inclemente el embate de todo tipo de proyectos de construcciones demandadas para el desarrollo del país y obligadas por la superpoblación que ha tenido lugar en las ciudades. Es innegable que esta superpoblación muy pronto se ha acomodado en cualquier espacio disponible para su supervivencia o multiplicación (Peñate, 2010).

Al igual que la teoría que hace referencia al hombre del estrecho de Bering (Haven, 1856), son muchas las teorías que fortalecen que la población buscó San Salvador ya sea para alejarse de otros peligros, buscar nuevos horizontes, huir de la guerra o para esconderse de depredadores que minaban su existencia. Sea cual sea la hipótesis, lo cierto es que no hay lugar en la capital salvadoreña en donde no se observe que el hombre y su instinto gregario han recibido el beso de la promesa de una ciudad para todos.

En esa dinámica, el ser humano ha construido su casa en donde le ha parecido más cómodo; en colonias nuevas que ofrecen muchos atractivos; en laderas que le han brindado distinción a su estilo de vida, a fuerza de la inclemencia con la que ha arremetido en contra del medio ambiente. Es innegable entonces que la preocupación por el resguardo ha sido más importante que la preservación de la tierra, despreocupándose de restituir la tierra, la madre de todo lo que existe, algo de lo que tan fácilmente le ha arrebatado. Al final, el ser humano, con su interferencia antropogénica, ha arrastrado inevitablemente a todos los que componen su núcleo familiar hacia ese destino con asomo de averno proclive al desencanto y a la destrucción: las cárcavas.

Las cárcavas representan un problema que exige reacción inmediata. No obstante, la indiferencia de las diferentes autoridades administrativas a las que les corresponde atender el fenómeno es evidente. Más dramático es darse cuenta de que los mismos pobladores que viven cerca de la zona deteriorada no hacen nada por mejorar el entorno. El hombre, preocupado por sus intereses inmediatos, ya no es aliado del medio ambiente, descubriendo de esa manera que el desgaste de la tierra es inminente.

La realidad siempre es visible. Es obvio el deterioro del ambiente en el país a causa de los diversos fenómenos que la embisten; agregando a ello que, además de no existir una educación ambiental adecuada, hay una falta de conciencia para conservar los ecosistemas, se sigue deteriorando de manera irremediable la superficie del suelo, cuyos mantos acuíferos y esencia de la biodiversidad se han ido extinguiendo por esa sed del hombre y su interferencia, que siempre quiere más.

\section{Definición, origen y formación de cárcavas}

Las cárcavas son una de las expresiones más evidentes de los procesos erosivos, sin embargo, son también de las geoformas menos estudiadas de modo sistemático. (GarcíaHidalgo, 2007); son zanjas que se forman en el suelo debido a diferentes causas, las cuales dañan al medio ambiente. Radulovich (1997) dice que son zanjas naturales causadas por el paso del agua, que socaba el suelo y lo arrastra. Rogers (2002) explica que las cárcavas son abarrancamientos formados en los materiales blandos por el agua de arroyos que, cuando falta una cobertera vegetal suficiente, ataca las pendientes excavando largos surcos de bordes vivos (ver figura 1).

Figura 1. Cárcava en la colonia Las Cañas. Alto: 16 m Kevin Coronado

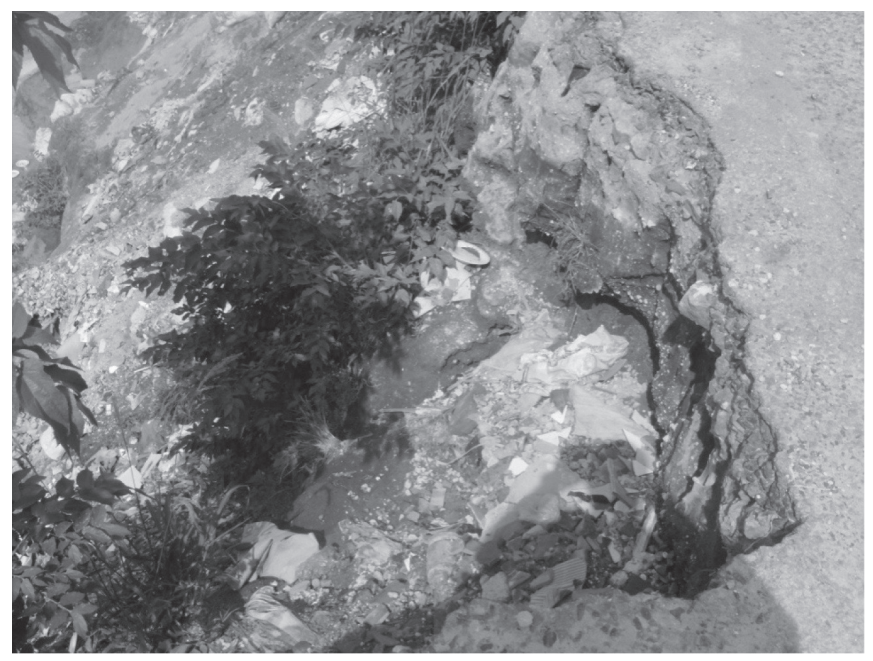

Fotografía tomada por equipo de investigación.

Conacher, A., \& Sala (1998) señalan que una cárcava es un conjunto de zanjas o conjuntos de canales que se desarrollan sobre capas de materiales no consolidados de la superficie terrestre. En la terminología internacional se les conoce como badlands. Pueden medir desde aproximadamente 30 
centímetros hasta varias decenas de metros de anchura. Al principio son surcos ramificados donde corre el agua, que progresivamente van creciendo de forma vertical, longitudinal y lateral hasta convertirse en torrentes (ver figura 2).

Figura 2. Cárcava en la colonia Altos del Boulevard.

Alto: 25 m Kevin Coronado

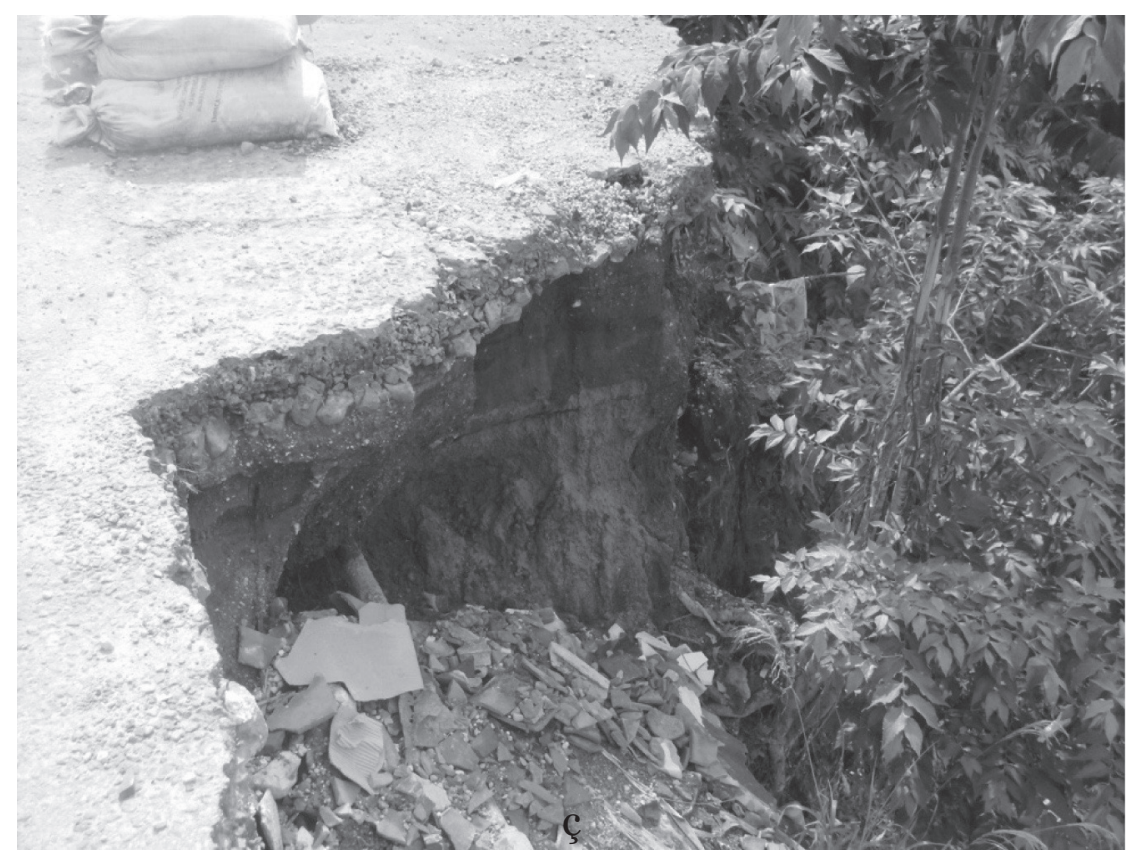

Fotografía tomada por equipo de investigación.

Las cárcavas o badlands aparecen con frecuen cia en el relieve arcilloso, pues la arcilla es una roca impermeable pero blanda. Martínez, Rubio, Oropeza, \& Palacios (2009) indican que, siendo producto de la erosión, las cárcavas generalmente siguen la pendiente máxima del terreno y constituyen un cauce natural en donde se concentra y corre el agua proveniente de las Iluvias, arrastrando gran cantidad de partículas del suelo. Los autores continúan diciendo que se inician cuando el suelo ha sido removido por el flujo superficial formando pequeños surcos, considerado como zanjeado incipiente; y a medida que aumenta el escurrimiento se forman pequeños canalillos que van creciendo en ancho y en profundidad hasta producir secciones transversales de diferentes formas, que se agrandan con la presencia de las avenidas máximas. Consecuentemente, las cárcavas se originan por la concentración de los escurrimientos superficiales en determinados puntos críticos del terreno (ver figuras 3 y 4 ). 
Figura 3. Cárcava ubicada en la colonia Las cañas 2010

(Zamora, 2010)
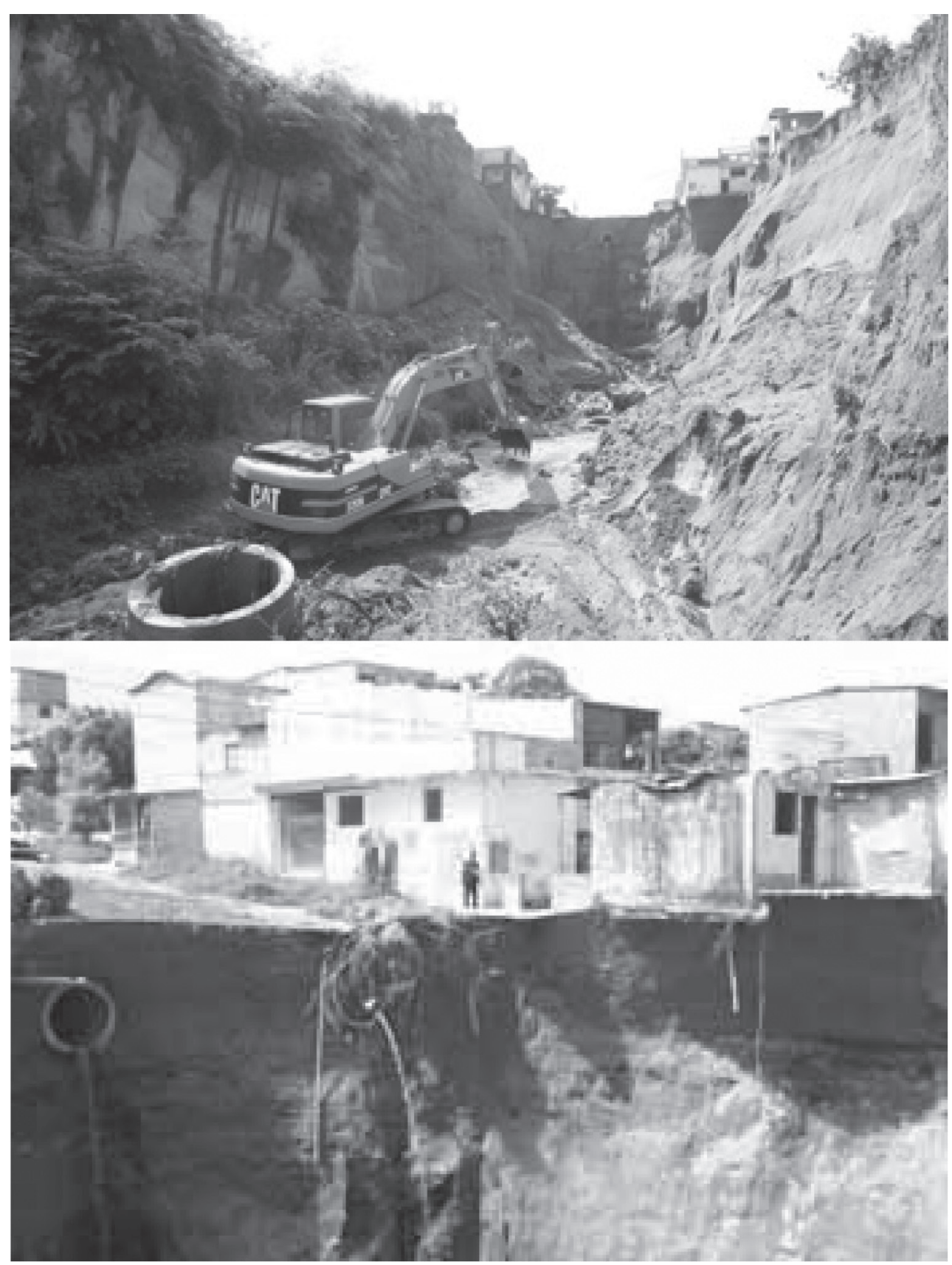

Fotografías tomadas por equipo de investigación. 
Figura 4. Cárcava de la colonia Las Cañas.

Alto: 16 m Kevin Coronado

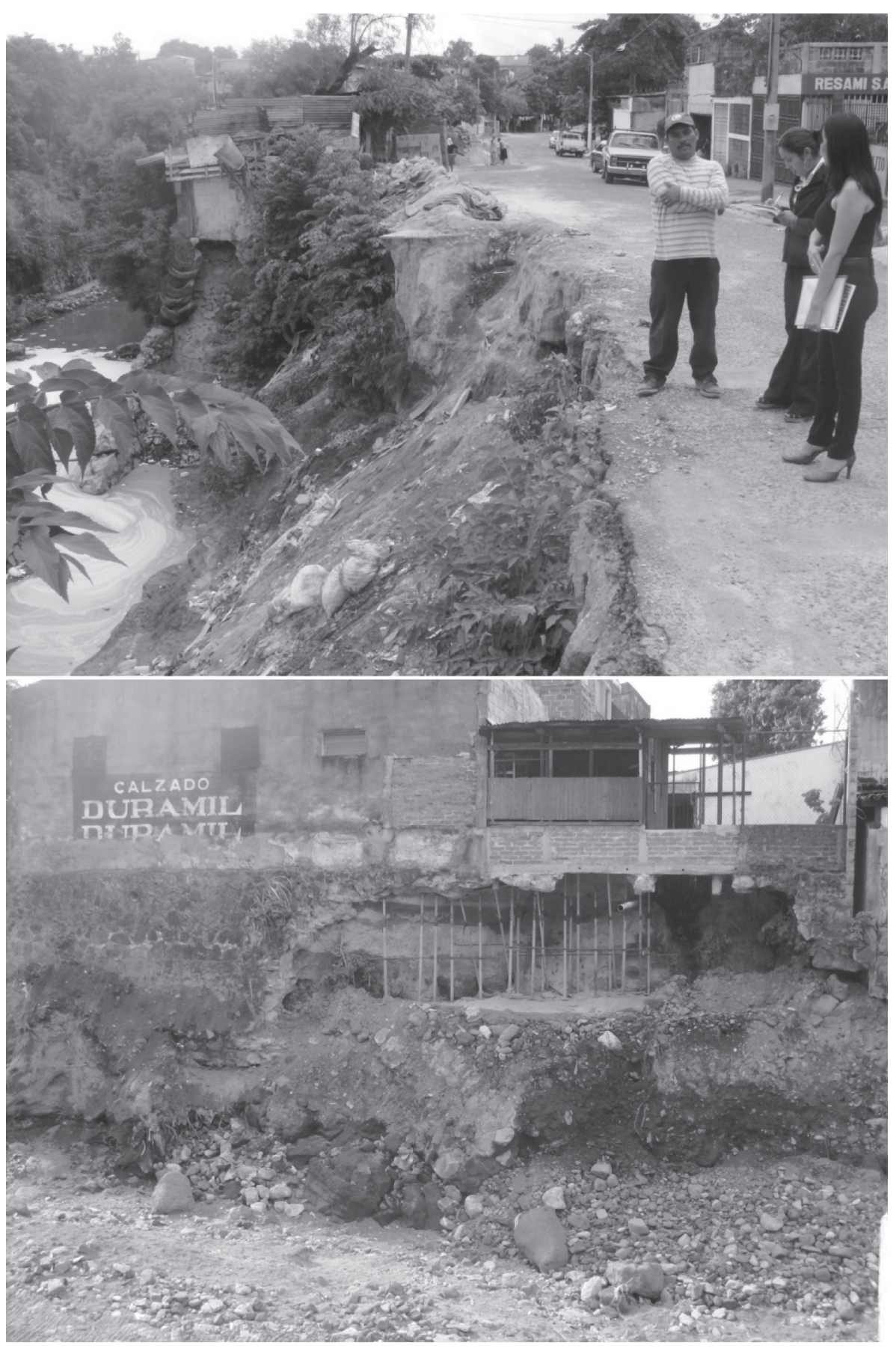

Fotografías tomadas por equipo de investigación. 
Las cárcavas son producto de la degradación biológica. La Convención de las Naciones Unidas para Combatir la Desertificación (CCD, 1994) define la degradación como la reducción o pérdida de la producción biológica y económica, así como de la complejidad de los ecosistemas terrestres, incluyendo suelos, vegetación, otros componentes biológicos, ecológicos y procesos geoquímicos e hidrológicos que operan en ellos (ONU, 1994).

Existen áreas extensas de tierras que están siendo sometidas a la degradación en forma irreversible, procesos de degradación como la erosión acelerada y la desertificación, compactación, acidificación, disminución en el contenido de materia orgánica, disminución de la biodiversidad genética y el agotamiento de la fertilidad natural del suelo (Posada, 2010).

Las cárcavas: el esfuerzo por comprender su incidencia y sanar las heridas de la tierra

Diversas investigaciones en torno al fenómeno de las cárcavas, y de cómo este se relaciona con el Estado, lentamente se han ido posicionando en el quehacer diario. Estudios como el de Calle, Rivera y Sinisterra (2007) proponen que la erosión por cárcavas son procesos de degradación que avanzan en forma acelerada como resultado del desconocimiento por parte de la sociedad sobre sus causas, consecuencias y soluciones. Peñate (2010) agrega que el problema de las cárcavas, especialmente en el área metropolitana de San Salvador, tiene a su base diferentes causas relacionadas con construcciones en lugares inadecuados, el mal manejo de aguas lluvias y negras y la falta de mantenimiento de drenajes.

Suárez, citado por Calle, Rivera y Sinisterra (2007), dice que las cárcavas se asocian con entregas localizadas de agua en sitios susceptibles. El aumento en la energía de las aguas desencadena un proceso localizado de erosión, donde los cultivos limpios en ladera, los surcos en sentido de la pendiente, el sobrepastoreo y la desprotección de los desagües son los principales factores que favorecen la formación de cárcavas.

Marelli (2004) agrega: “La erosión de cárcavas es un proceso de desgaste que ocurre naturalmente, no obstante, es casi siempre magnificado por la acción del hombre".

Sobre el suelo actúan básicamente los siguientes dos tipos de erosión:
- Geológica (a largo tiempo): incluye los procesos de erosión y formación de suelo en forma balanceada.

- Antrópica: la acción del hombre acelera los procesos erosivos debido a la eliminación de la vegetación natural, al exceso de laboreo, a la pérdida de nutrientes, etc.

La erosión es uno de los principales problemas de la agricultura en el mundo no solo porque afecta directamente el sitio donde ocurre, sino también por los efectos de sedimentación y contaminación que provoca. La pérdida de tierra cultivable en el mundo es estimada entre 5 y 7 millones de hectáreas por año (García-Hidalgo, 2007).

Las causas de la erosión del suelo son todavía poco conocidas. Aunque numerosas investigaciones, especialmente desde 1940, han dado como resultado una mejor comprensión de los procesos mecánicos de la erosión y su relación con el medio físico. Solo actualmente se han abordado investigaciones sistemáticas sobre los factores sociales, económicos, políticos e institucionales que actúan donde y cuando se produce la erosión (Morgan, 2009).

La persistencia de las lluvias, la tala de árboles, la mala forestación, los inadecuados tratamientos del suelo, el establecimiento indiscriminado de nuevos asentamientos humanos son solo algunos ejemplos de lo que no está contribuyendo a eliminar la erosión, sino que provocan la desertificación a gran escala. Es necesario comenzar a mejorar los ecosistemas y a fortalecer el suelo.

Para que el recurso del suelo esté adecuado a las necesidades de un ecosistema robusto, deben establecerse políticas que respondan a lograr los siguientes cuidados: a) disminuir a niveles aceptables la erosión del suelo (mayormente pérdidas de suelo por escorrentías o erosión hídrica) y b) proteger el suelo contra otros tipos de degradación, de las cuales las más comunes son la compactación, la disminución en fertilidad y reducciones en la capacidad de retención de agua (Radulovich, 1997).

En este contexto, se presenta la figura 5 en la que puede advertirse las dimensiones meteorológicas, ecológicas y humanas del problema, con propuestas de dinámicas corto y largo plazos, que deben ser abordadas en un futuro cercano en el umbral de una investigación dedicada al estudio exclusivo de las cárcavas y de la reconstrucción del suelo. 
Figura 5. La degradación del suelo en las zonas secas involucra interacciones complejas entre factores biofísicos (las dimensiones meteorológicas y ecológicas de la desertificación) y factores socioeconómicos (la dimensión humana de la desertificación). Fracasos pasados a la hora de reconocer el papel único de estas dimensiones y la interacción mediante varias escalas espaciales y temporales — cubriendo desde escalas locales hasta internacionales — han llevado a equivocaciones y controversias sobre el concepto (Reynolds \& Stafford, 2010).

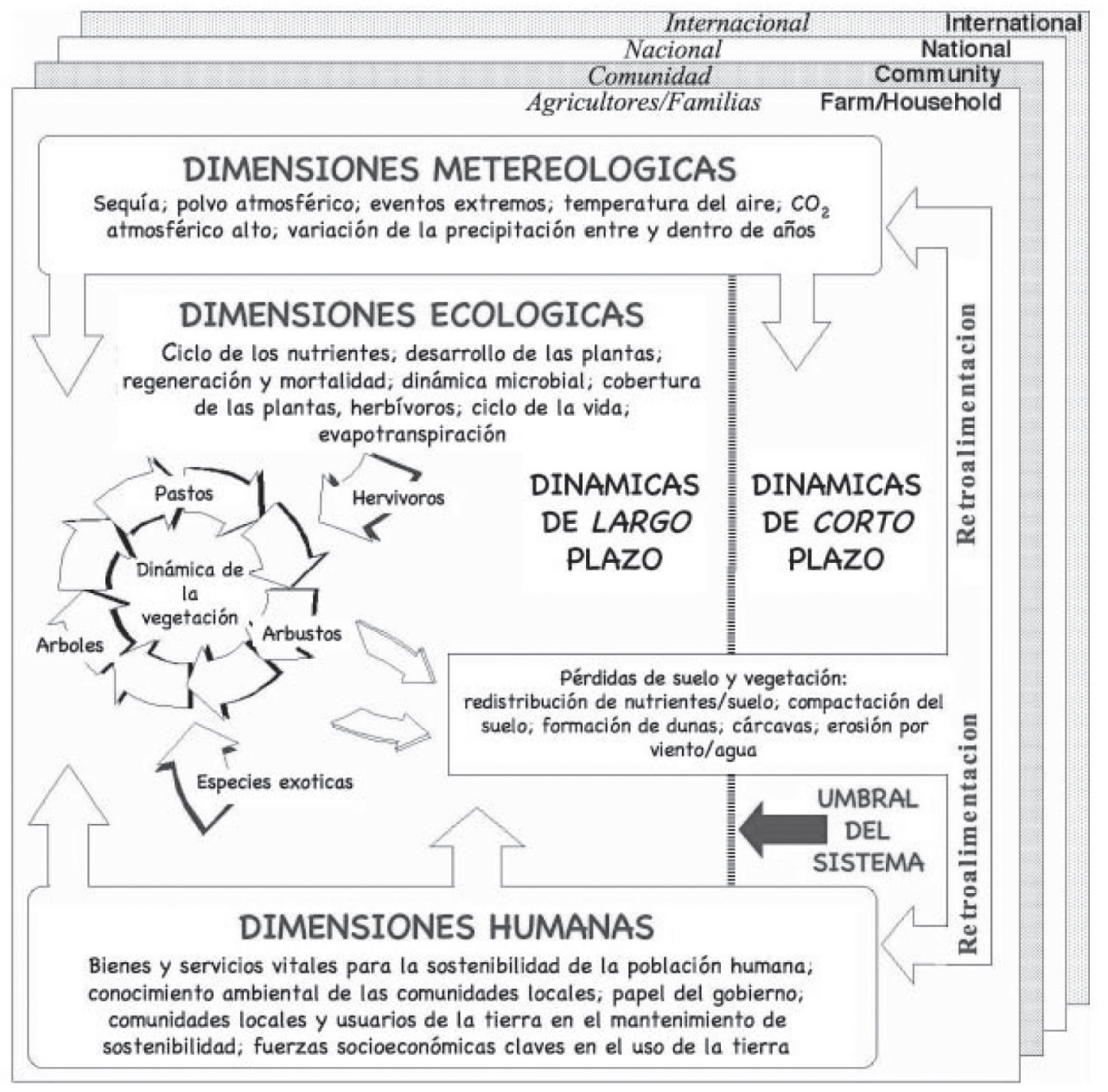

Este fenómeno forma parte del proceso natural de formación de la morfología del cauce y sus llanuras de inundación, y por tanto no debe ser impedido, sino tenido en cuenta a la hora de establecer el suelo urbano. (MOP, 2006)

\section{Problemas que están sobre la mesa}

López (2010) aporta a este estudio el siguiente análisis:

El fenómeno de las cárcavas en el país no es nuevo. El problema se agudizó en los últimos cinco años, debido a la expansión urbanística, a los daños en el sistema de drenajes de la ciudad y a las condiciones volcánicas del suelo, según coinciden expertos del Ministerio de Obras Públicas (MOP), la Asociación Salvadoreña de Ingenieros y Arquitectos (Asia) y varios consultores privados.

Las autoridades del MOP han identificado 132 cárcavas a escala nacional; 112 (80 \%) están en la zona metropolitana de San Salvador, específicamente en Ilopango, Soyapango, San Martín y en el sur de la capital.

Bonilla (2010) señala que en El Salvador había un subregistro del problema, es decir, solo se sabía de la existencia de 22 cárcavas, pero las tragedias de los últimos años por las torrenciales Iluvias, más la interacción con las comunidades 
afectadas y la verificación de los técnicos del MOP, han hecho que el número inicial de barrancos se multiplicara por seis.

Continúa asegurando Bonilla (2010) que, de la totalidad de estos, 32 están situados en las riberas de los ríos Acelhuate y Las Cañas. Ambos drenajes naturales ubicados en la zona metropolitana de San Salvador atraviesan municipios populosos. Por ejemplo, Acelhuate cruza por el sur de San Salvador, Ciudad Delgado, Apopa y Aguilares, entre otros. Mientras que el río Las Cañas comienza en Ilopango y se une con el Acelhuate en Apopa.

López (2010), enfatiza agregando, que un informe de la ONU reveló que el 95 por ciento de la población salvadoreña reside en zonas consideradas de alto riesgo, afirmó el viceministro de Obras Públicas, José Roberto Góchez.

El presidente de Asia, Juan Guillermo Umaña, indicó que el haber construido sin dejar las distancias de retiro de los ejes de las quebradas pone hoy en evidencia la vulnerabilidad de muchagente que vive en los alrededores. "Lamentablemente, durante muchos años no hemos podido detener el crecimiento urbano en zonas inseguras y se han cometido errores que ahora son evidentes por los deslizamientos y las cárcavas, que se deben a la falta de mantenimiento de los sistemas de aguas lluvias y aguas negras".

Los urbanizadores de vivienda de interés social también contribuyeron a generar el problema, porque en sus proyectos no desarrollaron la infraestructura necesaria para ingresar al drenaje natural (río o quebrada) sin afectar de manera considerable sus márgenes o causar erosión. Todo por su alto costo (López, 2010).

Umaña (2010) indicó que la falta de institucionalidad y aplicación de la legislación sobre urbanismo pone en evidencia la responsabilidad del Gobierno, ya que este debe velar por la seguridad de quienes residen en dichas zonas. Asegura también que el alto costo de las obras de mitigación se saldría del presupuesto del Gobierno, dado que reparar cada cárcava tiene un costo superior a los 500 mil dólares. A su vez, manifestó que la problemática de la deformación del suelo (cárcavas) se debe a la sobresaturación del sistema de drenajes en las principales ciudades.

Melara (2010) asevera que los sistemas de drenajes del gran San Salvador, construidos entre los años 30 y 40, no fueron pensados ni planeados para la enorme explosión demográfica de los últimos años. En San Salvador, por ejemplo, se calcula que residen más de dos millones de personas, casi la tercera parte de la población de todo el país. Esta densa población se ha asentado en zonas, en algunos casos, no aptas para el desarrollo urbanístico, lo que ha repercutido en la falta de capacidad de absorción del suelo durante las precipitaciones. Además, la insuficiencia de las alcantarillas y de los cauces de ríos y quebradas ha generado que las aguas lluvias se desborden sobre las carreteras, terrenos aledaños y laderas, generando problemas de erosión y daños en la red vial.

Melara (2010) considera que si a esto le sumamos que el suelo del área metropolitana está formado por ceniza volcánica joven o blanca, no es extraño que se generen grandes erosiones $y$, por ende, aparezcan enormes barrancos. Desde luego, el fenómeno se agudiza por la pendiente descendente en que se encuentra la zona metropolitana del país, desde El Picacho del volcán de San Salvador hasta el lago de llopango. "Cuando se construye sin control en el poniente de San Salvador, se comienza a alterar el piso y se vuelve impermeable el suelo, lo que ocasiona una gran escorrentía que al no tener donde filtrarse busca correr por la pendiente, lo que incrementa los caudales, la velocidad y fuerza de los ríos y quebradas".

López (2010) abona diciendo que la población no está capacitada ni organizada para inspeccionar y evaluar las condiciones de las desembocaduras de los colectores, lo que hace más grande el problema.

\section{Método}

La muestra estuvo conformada por 100 habitantes de las colonias más vulnerables de San Salvador y Soyapango, las que incluyeron las colonias Málaga, Las Cañas, Las Victorias y Altos del Boulevard, escogidas al azar mediante muestreo aleatorio, de un universo de 3.000 personas, habiéndose calculado la muestra con la fórmula para poblaciones finitas, correspondiendo su tamaño a un nivel de confianza del $90 \%$, con un error de estimación del $6 \%$. Respecto a los datos sociodemográficos de la muestra, el $42 \%$ fue del sexo masculino y el $58 \%$ del femenino; el $56 \%$ trabajaba, mientras el $44 \%$ no disponía de un empleo fijo.

Los instrumentos que se utilizaron para la recolección de datos fueron de tipo entrevista y cuestionario, ambos 
de elaboración propia. La entrevista fue de tipo enfocado con grupos de familias afectadas por la problemática, buscando obtener información complementaria mediante las reacciones de los entrevistados en los grupos focales realizados. Como complemento, se utilizó un instrumento de preguntas cerradas conformado por diez ítems, el que fue aplicado según la clasificación de la muestra determinada.

Para definir el tipo de estudio, se utilizó la clasificación propuesta por Montero y León (2007), escogiéndose el estudio descriptivo mediante encuestas, ya que se utilizarán análisis descriptivos para complementar el análisis de las entrevistas, las cuales fueron procesadas con el programa QSR, mientras que los datos cuantitativos se procesaron mediante el SPSS.

\section{Hallazgos}

El Estado es, en gran parte, responsable debido a que, si bien ha orientado la letra de las leyes para que desde su sustantividad se acusen con vigencia los delitos ambientales, no ha articulado un sistema que vele porque se cumpla. Uno de los principios de Derecho ambiental señalados en la Conferencia de Río dice que el que contamina paga. En el Código Penal, los artículos 255 al 263 establecen algunos parámetros para la protección del medio ambiente, pero no sirven de nada porque las leyes existen pero su aplicación aún no ve la luz.

El Estado no está poniendo suficiente atención e interés a estos problemas. No está aplicando la legislación a efecto de garantizar un desarrollo sostenible, tal como sugiere el informe Brundtland (ONU, 1987), para garantizar a las futuras generaciones un ambiente sano $y$, por lo tanto, la vida y el derecho a una vivienda digna, que es una de las garantías constitucionales vulneradas con la formación de cárcavas en las colonias no solo de San Salvador, sino de todo el territorio.

El Estado, en consecuencia, no está protegiendo las garantías constitucionales de los habitantes de El Salvador, especialmente de los que viven a orillas de las cárcavas.

Cotejando los hallazgos con los principios estudiados en la asignatura de Derecho Ambiental, se puede señalar que no solo el Estado es responsable del deterioro ambiental, por su falta de políticas, entre otros, sino que todos los habitantes de El Salvador deben compartir la responsabilidad —que es de todos- porque la letra de la ley está escrita para aplicarla, puesto que el Art. 8 del Código Civil dice que "nadie puede alegar ignorancia de ley" (Civil, 1860); para adherirla a la posibilidad de transformar un país. Todos son responsables: desde el que tira al suelo una bolsita vacía de churritos hasta aquel que directamente tala el oxígeno del mañana.

Queda al descubierto que muchas de las cárcavas que se forman en las colonias de San Salvador son producto de la deforestación.

Si bien las leyes están escritas, promulgadas y publicadas, no existe un sistema que exija su uso, permitiendo con ello el mal uso de los pocos recursos naturales que le quedan al territorio, lesionando así el medio ambiente.

Desde la perspectiva de la incidencia de las cárcavas, no existe suficiente evidencia del cumplimiento de los artículos 2, 117, 119 de la Constitución, quedando de manifiesto la no protección a las garantías que procura en materia de un medio ambiente sano y una vivienda digna.

\section{Discusión}

En El Salvador es innegable la crisis ambiental, la cual es un grave problema causado por factores como el desarrollo urbanístico, la fuerte migración interna y externa, los dramáticos cambios en la economía; yotros como el patrón de desarrollo del agro, la dinámica demográfica, el crecimiento poblacional de la periferia capitalista y la concentración en la tenencia de la tierra, que no son exclusivos de países que aún son considerados subdesarrollados, como los centroamericanos, ya que también los países altamente desarrollados han tenido que enfrentar el problema de la contaminación ambiental, debido a las exigencias que impone el mundo moderno con los avances tecnológicos de la ciencia y sus aplicaciones tecnológicas, siendo entonces en las naciones desarrolladas donde la problemática ambiental se siente con más intensidad por la competitividad que existe en ir hacia adelante en el desarrollo en toda su extensión. (Rosenda Artiga, 1998).

La falta de una legislación específica en El Salvador, aunado a que no existen tribunales especializados que hagan valer la letra de la ley, causará más condiciones para que el colapso del territorio sea inminente. 
Es necesario que las universidades alcen la voz y comiencen a pronunciarse frente a este fenómeno. Estudios geológicos, hidrológicos, de cárcavas subterráneas, de aire, etc., serían algunos de los instrumentos que coadyuvarían al Estado a cumplir con la tarea; y que abonarían a las soluciones que necesita el país.

Si bien la sobrepoblación ha agravado aún más su vulnerabilidad, el estar construyendo viviendas en terrenos inhabitables pone en perspectiva el incumplimiento de la norma constitucional.

En estos momentos, el problema de las cárcavas ya no se resolverá con la promulgación de nuevas leyes, o con el establecimiento de nuevas herramientas administrativas que ayuden en la vigilancia de los recursos naturales, o con nuevos puntos de vista jurídicos, sino con un cambio de actitud radical de todos los habitantes de El Salvador, grandes y pequeños, políticos y vendedores, maestros y sacerdotes, padres e hijos, frente al cuido de los recursos naturales, constituyendo una promesa para un mejor medio ambiente en este país, que tanto lo necesita.

\section{Referencias}

Arguello, F. A. (1994). Crisis de los Recursos Renovables y del Medio Ambiente en el Salvador. San Salvador.

CCD. (17 de Junio de 1994). Convención de las Naciones Unidas de Lucha contra la Desertificación, aprobada en París. Recuperado el 17 de Febrero de 2012, de Desertificación: http://www.cricyt.edu.ar/ enciclopedia/terminos/Deserti.htm

Civil, C. (1860). Código Civil. Decreto Ley №: S/N Fecha:23/08/1859 : Organo Legislativo.

Conacher, A., \& Sala, M. (1998). Las causas de degradación terrestre. Editorial John Wiley \& Sons Ltd.

Constitución (30 de Septiembre de 2011). Asamblea Legislativa de El Salvador. Constitución de la República de El Salvador. Recuperado el 16 de Marzo de 2012, de Centro de documentación Legislativa: http://www.asamblea.gob.sv/eparlamento/indicelegislativo/buscador-de-documentos-legislativos/ constitucion-de-la-republica/

Cuadra, S. (2010, 17 de julio). La lluvia en el gran San Salvador. El Diario de Hoy. 37.

Escalante, D (2010, julio). (Entrevista con Néstor Bonilla, Director de Planificación del Ministerio de Obras
Públicas: Los daños por lluvias suman \$ 322 millones.). Notas.

Funes, M. (17 de julio de 2009). El Salvador: la violación del derecho a la vivienda niega la posibilidad de una vida digna. Recuperado el 14 de Abril de 2012, de Discurso del Señor Presidente de la República Don Mauricio Funes sobre el acto de lanzamiento del "Programa global de vivienda y soluciones habitacionales pronunciado a través de cadena nacional, el día jueves 16 de julio de 2009.: http://raoulhernndezmyblog.wordpress. com/2009/07/17/el-salvador-la-violacion-del-derechoa-la-vivienda-niega-la-posibilidad-de-una-vida-digna/

García-Hidalgo, J. (6 de Agosto de 2007). Cárcavas cuaternarias. Obtenido de Página de José F. GarcíaHidalgo: http://www2.uah.es/jose_f_garcia_hidalgo/ investigacion/carcavas.htm

Haven, S. (1856). Archaelogy of the United States. . Washington DC: Smithsonian Institute.

Juárez, M. (2010). La erosión hídrica. Recuperado de http:// agrolluvia.com/wp-content/uploads/2010/02/intamarcos-juarez-suelos-la-erosion-hidrica.pdf

León, J. (12 de Abril de 2001). Estrategias para el control y manejo de la erosión de cárcavas. Recuperado el 2 de Febrero de 2012, de Unalmed.edu.co: http:// www.unalmed.edu.co/ poboyca/documentos/ documentos1/documentos-Juan\%20Diego/PInaifi_ Cuencas_Pregrado/Control\%20erosi\%F3n\%20 en\%20c\%E1rcavas\%20cuadernos\%20ambiental.pdf

López, J. (2010). San Salvador se hunde entre cárcavas. El Diario de Hoy , pág. 1.

Martínez, M., Rubio, E., Oropeza, J., \& Palacios, C. (2009). Control de cárcavas. SAGARPA, Secretaría de agricultura, ganadería, desarrollo rural, pesca y alimentación. México , 1-9.

Melara, E. (2010, julio). (Entrevista con agente de la Policía Nacional Civil Carlos Antonio Rodríguez y Geólogo Enrique Melara del SNET. Miles aislados. O. Servellón, \& J. López,entrevistadores). Notas.

Montero, I., \& León, O. (2007). A guide for naming research studies in psichology. International Journal of Clinical and Health Psychology , 847-862.

(MOP), M. D. (2006). Evaluación de inestabilidad de terrenos por asentamientos con urbanizaciòn deficitaria en el área Metropolitana de San Salvador - El Salvador. Problemática asociada a las inestabilidades del Terreno. San Salvador.

Morgan, Roy P.C. ( 1997) Erosión y Conservación del Suelo, Página 25 
ONU. (11 de Diciembre de 1987). Informe Brundtland "Nuestro futuro común", linforme de la Comisión Mundial sobre el Medio Ambiente y el Desarrollo (Resúmenes). Recuperado el 5 de Enero de 2012, de UN División de Desarrollo Sostenible, Departamento de Asuntos Económicos y Sociales: http://daccessdds-ny.un.org/doc/RESOLUTION/GEN/NRO/521/05/ IMG/NR052105.pdf?OpenElement

Peñate, S. (2010, 3 de julio). Las cárcavas revelan los errores urbanísticos. Diario El Mundo. 27

Posada, H. (2010). Fundamentos de la restauración ecológica. Recuperado el 12 de Marzo de 2012, de La web de la bioingeniería y la restauración ecológica: http:// ecoambientes.tripod.com/principios.html

Ramírez, C. (23 de Noviembre de 2010). La vivienda: una necesidad y un derecho humano. Recuperado el 12 de Febrero de 2012, de Noticias UCA YSUCA: http:// www.uca.edu.sv/noticias/nota.php?texto $=90798$

Reynolds, J., \& Stafford, M. (12 de Febrero de 2010). ¿Puede la humanidad causar desiertos? Recuperado el 2012 de 30 de Marzo, de ARIDnet: A Research Network for Studies of Global Desertification.
What is desertification?: http://www.biology.duke. edu/aridnet/pdfs/Dahlem\%20Book\%20Chaps/01Reynolds_sp.pdf

Rivera, J., Sinisterra, J., \& Calle, Z. (2002). Restauración ecológica de suelos degradados por erosión en cárcavas en el enclave xerofítico de Dagua, Valle del Cauca, Colombia. Centro para la investigación en sistemas sostenibles de producción agropecuaria CIPAV , 1-10.

Rosenda Artiga, S. C. (1998). Situación de la legislacion nacional e internacional vigente al adecuador control del desarrolo urbanistico del área metropolitana de San Salvador y su incidencia en el ambiente. San Salvador.

Umaña, J. (2010, julio). (Entrevista con Néstor Bonilla, Jefe de Planificación del MOP: El Salvador se hunde entre cárcavas. J. López, entrevistador). Notas.

Valladares, D. (2011, 02 de noviembre). Con derecho, pero sin techo. Diario Digital Contrapunto. 24.

Zamora, M. (19 de Junio de 2010). Familias afectadas por cárcava en Las Cañas trasladan pertenencias. La Prensa Gráfica. 